\title{
Pseudomonas aeruginosa as a Cause for Urinary Tract Infection in Children with No Predisposing Factors
}

\author{
Jithin C Shaji ${ }^{1}$, Remesh Kumar ${ }^{2}$
}

\begin{abstract}
Pseudomonas is considered to be an unusual organism in urinary tract infections. Most of the time, predisposing factors are present, which makes its colonization easier. In our case report, we conclude the possibility of asymptomatic colonization of Pseudomonas aeruginosa in urine even without any risk factors for its growth. So far, Pseudomonas urinary infection has been associated in children with urologic abnormalities or those who were hospitalized or catheterized or used any prior antibiotics. Following this case report, clinicians should note its unusual occurrence without any causative factors.

Keywords: Case report, Children, Community acquired, Pseudomonas aeruginosa, Risk factors, Urinary tract infection.

Pediatric Infectious Disease (2021): 10.5005/jp-journals-10081-1292
\end{abstract}

\section{BACKGROUND}

Urinary tract infection (UTI) is a very common illness in children. It can virtually occur in any age from neonates to adults. Properly taken sample for urine culture plays an important role in identifying the microorganism responsible for urinary infection. Moreover, it needs timely attention, appropriate evaluation, and treatment to cure the disease as recurrent infection can damage the renal parenchyma and cause hypertension and renal insufficiency later on in adulthood.

\section{Case Description}

An 8-month-old male child presented with history of high-grade fever of 1-day duration. No urinary symptoms were noted. Phimosis was absent on local examination. On systemic examination, focus for fever could not be found. Blood counts were within normal limits and CRP was negative. Urine was clear and routine test showed occasional pus cells only. Child was initiated on symptomatic treatment. On day 2, fever subsided. However, culture of a clean catch mid-stream sample done on 1st day showed heavy growth $\left(>10^{5}\right)$ of Pseudomonas aeruginosa. As he had no urinary symptoms and was afebrile by day 2 , repeat catheterized urine culture was sent on day 3 , which again showed heavy growth of Pseudomonas aeruginosa $\left(>10^{5}\right)$ with sensitivity to ceftazidime, aminoglycosides, and fluoroquinolones. He had no prior history of UTI, antibiotic usage, catheterization, or any other previous illnesses. The child was started on intravenous amikacin. His repeat urine culture after 5 days of treatment came as sterile. Ultrasonogram kidneys, ureters, and bladder showed normal anatomy of urinary system. Micturating cystourethrogram was done 6 weeks later and was found to be normal.

\section{Discussion}

Urinary tract infection is classically diagnosed by urine culture and sensitivity. The process of collecting urine from small infants can be quite tricky compared to a toilet-trained child. In children, urine is usually obtained by any of the four ways - sterile urine bag, clean catch, catheterization, or suprapubic aspiration. Sterile urine bag collection, though easy in infants, has got high contamination

\footnotetext{
1,2Department of Pediatrics, Apollo Adlux Hospital, Kochi, Kerala, India Corresponding Author: Jithin C Shaji, Department of Pediatrics, Apollo Adlux Hospital, Kochi, Kerala, India, Phone: +91 7592837693, e-mail: jithin15@yahoo.com
}

How to cite this article: Shaji JC, Kumar R. Pseudomonas aeruginosa as a Cause for Urinary Tract Infection in Children with No Predisposing Factors. Pediatr Inf Dis 2021;3(3):119-120.

Source of support: Nil

Conflict of interest: None

rates. Clean catch is the preferred mode of getting urine from older cooperative children. Catheterization is an invasive technique opted for young infants, that has contamination risks more or less similar to a clean catch sample. ${ }^{1}$ The gold standard is suprapubic aspiration which has very minimal contamination rates. However, this procedure is invasive and can evoke parent's anxiety. So, parental preference is another factor that determines the method of urine collection in children.

The most common pathogen in UTI is E. coli $(65-75 \%){ }^{2}$ Less likely ones include Klebsiella (23\%), Proteus (7\%), other Enterobacteriaceae, Enterococcus, Pseudomonas aeruginosa, and Staphylococcus saprophyticus (1-4\%). ${ }^{2,3}$ Various literatures have reported frequency of Pseudomonas urinary infection to be $2.4-9 \% .{ }^{4-6}$ One study reported the incidence of Pseudomonas UTI in healthy children in their population to be $6.7 \%{ }^{7}$ and described history of prior UTI, use of antibiotics, hospitalization, genitourinary anomalies, vesicoureteral reflux, and previous surgeries as potential causes for Pseudomonas colonization in urine. ${ }^{6,7}$ Here, we report a case of first episode of community-acquired Pseudomonas UTI in an infant who has no major risk factors for this infection.

Pseudomonas UTI is significant because of their innate resistance to common antibiotics used for urinary infections. Because of that, it is more often seen in children who received common antibiotics for uroprophylaxis as most oral antibiotics have no action against Pseudomonas, which favors its selective colonization. Literature mentioning community-acquired Pseudomonas urinary infection in children are scarce. A study by Ozumba ${ }^{4}$ in Nigeria showed that Pseudomonas was responsible 
mainly for hospital-acquired UTIs in children with urinary tract surgeries and other predisposing conditions. Other studies from Israel ${ }^{6}$ and Greece ${ }^{7}$ mentioned about children with communityacquired Pseudomonas UTI. Almost all literatures described the association of Pseudomonas to some type of urinary anomalies or prior antibiotic usage or other significant factors.

\section{Conclusion}

Unlike the past literature, our patient neither had any urological abnormalities nor had other predisposing factors. This suggests the possibility of asymptomatic colonization of Pseudomonas aeruginosa in the urinary tract of children in the community. Through this report, we want to make the practicing pediatricians aware of this rare possibility also.

\section{References}

1. Karacan C, Erkek N, Senel S, et al. Evaluation of urine collection methods for the diagnosis of urinary tract infection in children. Med Princ Pract 2010;19(3):188-191. DOI: 10.1159/000273068.
2. Spahiu L, Hasbahta V. Most frequent causes of urinary tract infections in children. Med Arh 2010;64:88-90.

3. Lo DS, Shieh HH, Ragazzi SL, et al. Community-acquired urinary tract infection: age and gender-dependent etiology. J Bras Nefrol 2013;35(2):93-98. DOI: 10.5935/0101-2800.20130016.

4. Ozumba UC. Urinary tract infections in children due to Pseudomonas aeruginosa in Enugu, Nigeria. J Trop Pediatr 1998;44(5):315. DOI: 10.1093/tropej/44.5.315.

5. Goldman M, Rosenfeld-Yehoshua N, Lerner-Geva L, et al. Clinical features of community-acquired Pseudomonas aeruginosa urinary tract infections in children. Pediatr Nephrol 2008;23(5):765. DOI: 10.1007/s00467-007-0697-6.

6. Marcus N, Ashkenazi S, Samra Z, et al. Community-acquired Pseudomonas aeruginosa urinary tract infections in children hospitalized in a tertiary center: relative frequency, risk factors, antimicrobial resistance and treatment. Infection 2008;36(5):421. DOI: 10.1007/s15010-008-7328-4.

7. Bitsori M, Maraki S, Koukouraki S, et al. Pseudomonas aeruginosa urinary tract infection in children: Risk factors and outcomes. J Urol 2012;187(1):260-264. DOI: 10.1016/j.juro.2011.09.035. 